\section{P3-S1.44 COMPARISON OF SELF-TAKEN VULVO-VAGINAL SWABS AND CLINICIAN-TAKEN ENDOCERVICAL SWABS USING THE GEN-PROBE APTIMA COMBO 2 ASSAY VS CLINICIAN- TAKEN URETHRAL AND ENDOCERVICAL SWABS FOR CULTURE OF GONORRHOEA}

doi:10.1136/sextrans-2011-050108.444

J Wilson, C Stewart, S Schoeman, R Booth, S Smith, M Wilcox. Leeds Teaching Hospitals NHS Trust, Leeds, UK

Background The role of non-invasive testing for gonorrhoea (GC) in women has not yet been fully established in the UK. Validation of NAATs in low prevalence populations has been recommended. Our study is the first to compare gonorrhoea detection on self-taken VVSs by AC2 assay with gold-standard culture of clinician-taken urethral and endocervical (endocx) samples. As it is unclear whether a VVS or endocx swab is better for the detection of chlamydia (CT) by AC2 assay in women needing an examination we also compared the sensitivities of these samples for CT detection.

Methods Women aged $16+$ requesting STI testing consenting to perform a self-taken VVS prior to routine examination were included. Clinicians took urethral and endocx samples for gonorrhoea culture and an endocx swab for AC2 assay. AC2 positives were confirmed with Aptima GC and Aptima CT assays.

Results 3973 women included, 100 (2.5\%) were infected with GC. Overall sensitivities were culture $82 \%$; clinician taken endocx AC2 96\%; self-taken VVS AC2 99\% ( $\mathrm{p}=0.0002)$. The specificity of all the AC2 tests was $100 \%$. In women with symptoms the sensitivities were culture 84\%; clinician taken endocx AC2 100\% and VVS AC2 $100 \%$ ( $p=0.003$ ). In women with no symptoms $1.9 \%$ had GC. The sensitivities were culture 79\%; clinician taken endocx AC2 91\% and self-taken VVS AC2 97.5\% ( $p=0.015)$. The endocx AC2 performed less well in women without symptoms, 91\% vs $100 \%(p=0.031)$; the VVS AC2 assay performed equally well, $97.5 \%$ vs $100 \%$ $(p=0.41)$. Overall sensitivities for CT were clinician taken endocx AC2 89\%; self-taken VVS AC2 97\% $(p=0.0001)$. In women with symptoms the sensitivities were clinician taken endocx AC2 88\%; self-taken VVS AC2 97\% ( $p=0.001)$. In women with no symptoms the sensitivities were clinician taken endocx AC2 89\%; self-taken VVS AC2 98\% ( $p=0.002)$.

Conclusion AC2 assay of self-taken VVSs was significantly more sensitive for the detection of GC than culture of urethral and endocx samples and equivalent to detection by AC2 assay from cliniciantaken endocx swabs. The specificity and PPV of the AC2 assay was very high in this low prevalence population. AC2 assay of self-taken VVSs was significantly more sensitive for the detection of CT than AC2 assay of clinician-taken endocx samples. On the basis of these findings a self-taken VVS is the sample of choice in women who do not need an examination. In those who are being examined either a self-taken or clinician-taken VVS is the sample of choice giving better detection rates of GC and CT than an endocx swab.

\section{Clinical sciences poster session 2: herpes simplex virus \\ P3-S2.01 NON SEXUALLY TRANSMITTED GENITAL ULCERS; PATIENTS REFERRED TO A STD CLINIC}

doi:10.1136/sextrans-2011-050108.445

\section{A Palit. BLDE University, Bijapur, India}

Introduction Non-sexually transmitted genital ulcers are a cause of significant morbidity among sexually active young men and women. Establishing the underlying cause of the genital ulcer and differentiation from sexually transmitted infections may be challenging for the treating physician.
Case reports Patient data were collected from a STD clinic in a tertiary care hospital in south India from 2008 to 2010. All the patients were routinely screened for syphilis and HIV infection. Smears from the ulcers were taken and stained with Gram stain and/or Giemsa stain. Biopsy from the ulcer edge was taken whenever indicated. Following causes for non-sexually transmitted genital ulcers were recorded: Behcet's disease was seen in two male patients. Infectious ulcers; One pregnant woman presented with multiple painful genital ulcers caused by Klebsiella spp. An HIV infected man on antiretroviral therapy presented with ecthyma gangrenosum of scrotum with unilateral lymphadenopathy caused by Pseudomonas sp. Chancroid-like ulcers; a monogamous, HIV-negative office worker and an HIV positive widow, sexually inactive since 5 years, presented with multiple, small shallow genital ulcers with excruciating pain simulating herpes genitalis. The ulcers did not respond to adequate therapy with acyclovir and organism could not be demonstrated on gram stain or bacterial culture. In both the cases, the ulcers healed completely with azithromycin. Factitious ulcer; seen over the shaft of penis in an unmarried man. Genital apthae due to chikungunya fever; during an epidemic of chikungunya fever in the region, 25 patients with acute disease presented with multiple aphthous ulcers involving scrotum, penoscrotal junction and adjacent crural region. Skin biopsy from the ulcers reveals lymphocytic vasculitis.

Conclusion Causes of genital ulcers in patients referred to STD clinic may be varied. Atypical cases must be examined with care to identify the cause. Counselling plays an important role in the management of patients with non-sexually transmitted genital ulcer.

\section{P3-S2.02 VARIATIONS IN TESTING AND TREATMENT RECEIVED BY INFANTS WITH POSSIBLE NEONATAL HERPES}

doi:10.1136/sextrans-2011-050108.446

${ }^{1} \mathrm{E}$ Meites, ${ }^{1} \mathrm{~F} \mathrm{Xu},{ }^{1} \mathrm{~K}$ Hutchins, ${ }^{2} \mathrm{~B}$ Ackerson, ${ }^{1} \mathrm{~J}$ Gee, ${ }^{3} \mathrm{E}$ Eriksen, ${ }^{4} \mathrm{~A}$ Naleway, ${ }^{1} \mathrm{~L}$ Markowitz, ${ }^{3} \mathrm{~K}$ Zangwill. ${ }^{1}$ Centers for Disease Control and Prevention, Atlanta, USA; ${ }^{2}$ Southern California Kaiser Permanente Medical Group, Los Angeles, USA; ${ }^{3}$ UCLA Center for Vaccine Research, Torrance, USA; ${ }^{4}$ Kaiser Permanente Northwest Center for Health Research, Portland, USA

Background Data are scarce on clinical care of infants with possible neonatal herpes simplex virus (HSV) infection, a rare and serious condition that should be treated with systemic acyclovir for 14-21 days. We reviewed HSV testing and treatment in a large cohort of US infants in order to assess clinical care received.

Methods We investigated $>270000$ infants born from 1997 to 2002 at three managed care organizations participating in the US Vaccine Safety Datalink. Medical records were abstracted if an infant had a discharge ICD-9 code compatible with HSV infection, a positive laboratory test for HSV, or neonatal death. Abstracted data included symptoms, testing, and treatment. Two physicians reviewed likely HSV infections. We identified confirmed cases (compatible symptoms and positive laboratory test), probable cases (compatible symptoms only), and others (with an alternate diagnosis). Descriptive frequencies were calculated.

Results We abstracted records from 770 infants, identifying 35 cases (24 confirmed and 11 probable) and 735 others. HSV infection manifested as skin, eye, and mucosal (SEM) disease in 20 cases, central nervous system (CNS) disease in 8 cases, and disseminated disease in seven cases. Among 35 cases, all 35 (100\%) had symptoms compatible with HSV infection; these included vesicular lesions in $20(57 \%)$ and seizure in 7 (20\%). Overall, 35 (100\%) were ever tested for HSV. At least 34 (97\%) received some acyclovir; median time to treatment, available for 32 cases, was 3 days (range 0-35 days). Only $8(23 \%)$ received systemic acyclovir for the recommended 